JOURNAL OF

SYMPLECTIC GEOMETRY

Volume 4, Number 2, 149-170, 2006

\title{
TRANSVERSE KNOTS, BRANCHED DOUBLE COVERS AND HEEGAARD FLOER CONTACT INVARIANTS
}

\author{
Olga Plamenevskaya
}

\begin{abstract}
Given a transverse link in $\left(S^{3}, \xi_{\text {std }}\right)$, we study the contact manifold that arises as a branched double cover of the sphere. We give a contact surgery description of such manifolds, which allows to determine the Heegaard Floer contact invariants for some of them. By example of the knots of Birman-Menasco, we show that these contact manifolds may fail to distinguish between non-isotopic transverse knots. We also investigate the relation between the Heegaard Floer contact invariants of the branched double covers and the Khovanov homology, in particular, the transverse link invariant we introduce in a related paper.
\end{abstract}

\section{Introduction}

Let $\left(S^{3}, \xi_{\text {std }}\right)$ be the 3 -sphere equipped with its standard contact structure $\xi_{\text {std }}=\operatorname{ker}(d z-x d y)$. A link $L \subset S^{3}$ is called transverse if it is everywhere transverse to the contact planes. Let $\Sigma(L)$ be the double cover of $S^{3}$ branched over $L$. Then, $\Sigma(L)$ carries a natural contact structure $\xi_{L}$ lifted from $\left(S^{3}, \xi_{\text {std }}\right)$. The goal of this paper is to study the contact manifold $\left(\Sigma(L), \xi_{L}\right)$.

Our motivation is two-fold. First, one might wonder whether the double branched covers can help us understand transverse knots and links. The classification of transverse knots is a very difficult task. Indeed, while a few simplest knots, such as the unknot, the figure eight knot, and torus knots are completely classified by their topological knot type and the selflinking number $[\mathbf{1}]$, in general this is not true. The first examples of smoothly isotopic, but not transversely isotopic transverse knots $K_{1}, K_{2}$ with the same self-linking number were given by Birman and Menasco [2]. The existence of such pairs was also demonstrated by Etnyre and Honda [3] via some "nonexplicit" examples. Unfortunately, it seems that the branched double covers do not capture the subtle difference between such knots. Indeed, we prove 
Theorem 1.1. Let $K_{1}, K_{2}$ be transversely non-isotopic knots of [2, Theorem 1.6]. The branched double covers $\left(\Sigma\left(K_{1}\right), \xi_{K_{1}}\right)$ and $\left(\Sigma\left(K_{2}\right), \xi_{K_{2}}\right)$ are contactomorphic.

(Theorem 1.6 in $[\mathbf{2}]$ provides a family of pairs of transversely non-isotopic knots, not just a single pair. Our result is true for all pairs of [2]).

From another viewpoint, double covers of $\left(S^{3}, \xi_{\text {std }}\right)$ branched over transverse links give an interesting special case of contact 3 -manifolds. Indeed, the works of Giroux [4] and others imply that every three-dimensional contact manifold can be represented as a triple branched cover of $\left(S^{3}, \xi_{\text {std }}\right)$. It turns out that branched double covers are quite simple. We give an algorithm for finding a contact surgery diagram $[\mathbf{5}, \mathbf{6}]$ for $\left(\Sigma(L), \xi_{L}\right)$; the diagrams we get only involve surgeries on Legendrian unknots. We are also able to find the homotopy invariants of the contact structure $\xi_{L}$ (that is, the induced $\mathrm{Spin}^{c}$ structure and the three-dimensional invariant $d_{3}(\xi)$ of $\left.[\mathbf{7}]\right)$.

Theorem 1.2. Let $\mathfrak{s}_{L}$ be the $\operatorname{Spin}^{c}$ structure induced by $\xi_{L}$. Then $c_{1}\left(\mathfrak{s}_{L}\right)=0$. The invariant $d_{3}(\xi)$ is completely determined by the topological link type of $L$ and its self-linking number $\operatorname{sl}(L)$.

In certain cases, it is easy to tell whether the contact structure $\xi_{L}$ is tight or overtwisted.

Proposition 1.3. $\left(\Sigma(L), \xi_{L}\right)$ is overtwisted if $L$ is obtained as a transverse stabilization of another transverse link.

Proposition 1.4. $\left(\Sigma(L), \xi_{L}\right)$ is Stein fillable if the transverse link $L$ is represented by a quasipositive braid.

(Here and later on, it will be convenient to represent transverse links as closed transverse braids in $\left(S^{3}, \xi_{\text {std }}\right)$. We give more details about this representation in the next section.)

We now turn attention to the Heegaard Floer contact invariants. The Heegaard Floer theory of Ozsváth and Szabó ([8] and sequels) associates a homology group $\widehat{\mathrm{HF}}(Y)$ to a closed oriented 3-manifold $Y$ and yields invariants for many low-dimensional objects. In particular, given a contact structure $\xi$ on $Y$, the contact invariant $c(\xi)$ is a distinguished element of $\widehat{\mathrm{HF}}(-Y)$, defined up to sign $[\mathbf{9}]$. (We assume that the coefficients are taken in $\mathbb{Z}$.)

Propositions 1.3 and 1.4 along with the surgery diagrams and properties of $c(\xi)$ enable us to determine the Ozsváth-Szabó contact invariant $c\left(\xi_{L}\right)$ for $\left(\Sigma(L), \xi_{L}\right)$ in many cases. We observe that the contact invariant $c\left(\xi_{L}\right)$ behaves very similarly to the Khovanov-homological invariant of transverse links that we introduce in [10]. This is not a mere coincidence. Indeed, as proved by Ozsváth and Szabó [11], for a smooth link $L \subset S^{3}$, there is a spectral sequence converging to $\widehat{\mathrm{HF}}(-\Sigma(L))$ whose $E^{2}$ term is given by the reduced Khovanov homology of $L$ (both theories are to be taken with $\mathbb{Z} / 2 \mathbb{Z}$ 
coefficients). When the link $L$ is alternating, the spectral sequence of $[\mathbf{1 1}]$ collapses at the $E^{2}$ stage, providing an isomorphism

$$
\widetilde{\mathrm{Kh}}(L) \cong \widehat{\mathrm{HF}}(-\Sigma(L)) .
$$

Now, suppose the link $L$ is transverse, and $\xi_{L}$ is the induced contact structure on the branched double cover. Let $\psi(L) \in \widetilde{\mathrm{Kh}}(L)$ be the invariant of [10]. We would like to suggest that the elements $\psi(L)$ and $c\left(\xi_{L}\right)$ correspond to one another under the isomorphism (1.1). However, we must be careful, because this isomorphism is not canonical: while the spectral sequence of [11] is believed to be an invariant of the link, this invariance has not been proved. To deal with this issue, we fix a link diagram before studying such an isomorphism. (The choice of the diagram will be clear from the context; besides, we prove that both $\psi(L)$ and $c\left(\xi_{L}\right)$ are independent of the diagram.)

We also need to be more precise about the spectral sequence and isomorphism (1.1). For a fixed link diagram, the construction of [11] gives a filtered chain complex $C(L)$, whose homology is $\widehat{\mathrm{HF}}(-\Sigma(L))$, and the associated graded complex is the chain complex $\widetilde{C K h}(L)$ for reduced Khovanov homology (with its homological grading). When $L$ is alternating, the spectral sequence collapses, yielding a canonical isomorphism between $\widetilde{\mathrm{Kh}}(L)$ and the associated graded group of $\widehat{\mathrm{HF}}(-\Sigma(L))$. For coefficients in a field, the associated graded group of $\widehat{\mathrm{HF}}(-\Sigma(L))$ is of course isomorphic to $\widehat{\mathrm{HF}}(-\Sigma(L))$, but the latter isomorphism is not canonical. Therefore, we will always think of $(1.1)$ as the isomorphism between $\widetilde{\mathrm{Kh}}(L)$ and the associated graded group of $\widehat{\mathrm{HF}}(-\Sigma(L))$. The relation between $\psi(L)$ and $c\left(\xi_{L}\right)$ must involve gradings, as follows. Recall from [10] that $\psi(L)$ is a homogeneous element of $\widetilde{\mathrm{Kh}}(L)$ of homological degree 0 . Let $c_{0}\left(\xi_{L}\right)$ be the image of $c\left(\xi_{L}\right)$ in the corresponding subquotient of the associated graded group of $\widehat{\mathrm{HF}}(-\Sigma(L))$, that is, $c_{0}\left(\xi_{L}\right) \in \widehat{\mathrm{HF}}_{0}(-\Sigma(L)) / \widehat{\mathrm{HF}}_{1}(-\Sigma(L))$, where the subscripts on $\widehat{\mathrm{HF}}$ indicate the filtration level.

We suggest

Conjecture 1.5. If $L$ is a transverse representative of an alternating smooth link, then the homological grading of $\psi(L) \in \widetilde{\mathrm{Kh}}(L)$ is the same as the filtration level of $c\left(\xi_{L}\right) \in \widehat{\mathrm{HF}}(-\Sigma(L))$ (and this filtration level is 0 ). Moreover, $\psi(L)=c_{0}\left(\xi_{L}\right)$ under the isomorphism (1.1) between $\widetilde{\mathrm{Kh}}(L)$ and the associated graded group of $\widehat{\mathrm{HF}}(-\Sigma(L))$.

In the general case, it is plausible that $c\left(\xi_{L}\right)$ somehow "corresponds" to $\psi(L)$ under the spectral sequence.

In the special case when the transverse link $L$ is represented by a transverse closed braid whose braid diagram is alternating, Conjecture 1.5 is not hard to prove. 
Theorem 1.6. Let $L$ be a transverse link represented by a closed braid with an alternating braid diagram. Then, the filtration level of $c(\xi)$ is as stated, and $\psi(L)=c_{0}\left(\xi_{L}\right)$.

It should be noted that alternating braids represent a very narrow class of links. We show that $\psi(L)=c\left(\xi_{L}\right)=0$ for all such links except the $(2, n)$-torus link.

Conjecture 1.5 implies that $c\left(\xi_{L}\right) \neq 0$ whenever $\psi(L) \neq 0$. We are able to check this directly for many cases not covered by Theorem 1.6 (see Section 6). If proved in general, Conjecture 1.5, together with the fact that $c(\xi)$ vanishes when $\xi$ is overtwisted [9], would give a powerful sufficient condition for the contact structure $\xi_{L}$ to be tight. Indeed, for an arbitrary transverse link $L$, we can often show that $\psi(L) \neq 0$ by using arguments from [10] or software $[\mathbf{1 2}, \mathbf{1 3}]$; when $K$ is an alternating knot, it can be shown that $\psi(K) \neq 0$ if and only if $\operatorname{sl}(K)=-\sigma(K)-1$, where $\sigma(K)$ is the signature of the knot (with the sign convention such that the right-handed trefoil has signature -2.) Note that the contact structures that we would thus obtain from Theorem 1.6 are all (trivially) Stein fillable.

\section{Transverse links and braids}

In what follows, we will be working with the induced contact structure $\xi_{L}$ on the branched double cover $\Sigma(L)$ for a transverse link $L$ in the standard contact 3 -sphere. We now describe in some detail how $\xi_{L}$ is constructed.

Let $L$ be a transverse knot in $S^{3}$ (if $L$ is a link, we can deal with every component separately). Then some neighborhood of $L$ embeds into $\mathbb{R}^{2} \times S^{1}$ via coordinates $(r, \theta, z)$ (with $(r, \theta)$ the polar coordinates on $\mathbb{R}^{2}, z \in S^{1}$, and $L=\{r=0\}$ ), and the contact structure $\xi$ in this neighborhood can be given as the kernel of the 1 -form $d z+r^{2} d \theta$. (This is the Darboux theorem for contact structures.) In this neighborhood, the standard local model for the projection $p: \Sigma(L) \rightarrow S^{3}$ is given by the map $(w, z) \rightarrow\left(w^{2}, z\right)$, where $w=x+i y$.

Fix $n$ large enough, so that the set $\left\{r=r_{0}\right\}$ with $r_{0}^{4}=\frac{1}{4 \pi n}$ is contained in the chosen neighborhood. Choose $\eta, \epsilon>0$ such that $\eta<r_{0}$, and $\epsilon^{2}<2 \eta^{4}$. Define $\xi_{L}$ on $\Sigma(L)-\{r<\eta\}$ to be the kernel of the pull-back contact form (i.e., $\xi_{L}=\operatorname{ker}\left(d z+2 r^{4} d \theta\right)$ where our coordinates are defined, and $r \geq \eta$ ). For $r<\epsilon$, let $\xi_{L}$ be the kernel of the contact form $d z+r^{2} d \theta$, and interpolate between the two pieces by setting $\xi_{L}=\operatorname{ker}(d z+f(r) d \theta)$, where the smooth function $f$ is chosen so that $f(r)=r^{2}$ for $r<\epsilon, f(r)=2 r^{4}$ for $r>\eta$, and $f^{\prime}(r)>0$ for $r>0$. It is clear that $d z+f(r) d \theta$ is a contact form; moreover, the contact structure it defines inside the coordinate neighborhood of $L$ in $\Sigma(L)$ is isotopic to $\operatorname{ker}\left(d z+r^{2} d \theta\right)$ and therefore tight. (Note that the pullback form $d z+2 r^{4} d \theta$ would not work for the entire $\Sigma(L)$ : this form is not contact along the $z$-axis.) 
We have to check that the contact structure $\xi_{L}$ we obtain is independent of choices. To this end, observe that the characteristic foliation that $\xi_{L}$ induces on the torus $\left\{r=r_{0}\right\}$ is given by parallel longitudes of framing $-n$ (calculated with respect to the framing defined by $\theta$ ). Fix two of these parallel longitudes. They divide the torus $\left\{r=r_{0}\right\}$ into two annular regions; by pushing one of these regions in and another one out, we can perturb this torus by an isotopy into a convex surface whose characteristic foliation is Morse-Smale, and the dividing set is given by the two parallel longitudes of framing $-n$. In addition, we can assume that the support of this isotopy lies outside the set $\{r \leq \eta\}$. We denote the new convex torus by $T_{n}$ and the tubular neighborhood of $L$ in $\Sigma(L)$ that $T_{n}$ bounds by $V_{n}$.

With given boundary conditions, the tight contact structure in the solid torus bounded by $T_{n}$ is unique up to an isotopy (see, e.g., [14]). This means that the tight contact structure on $V_{n}$ is uniquely determined by the boundary conditions, and, since our construction of $\xi_{L}$ outside of $T_{n}$ is canonical, the contact structure $\xi_{L}$ on $\Sigma(L)$ is independent of all choices (including the choice of $n$ ).

We will need a few more facts about transverse knots. (See [15] for a detailed survey.)

It will be helpful to think about transverse links by representing them by closed braids. For this, consider the symmetric version of $\left(S^{3}, \xi_{\text {sym }}\right)$ with $\xi_{\mathrm{sym}}=\operatorname{ker}(d z+x d y-y d x)$. Then, any closed braid around $z$-axis can be made transverse to the contact planes; moreover, any transverse link in $\left(S^{3}, \xi_{\text {std }}\right)$ is transversely isotopic to a closed braid [16].

To define the self-linking number $s l(L)$, trivialize the plane field $\xi$, and let the link $L^{\prime}$ be the push-off of $L$ in the direction of the first coordinate vector for $\xi$. Then, $\operatorname{sl}(L)$ is the linking number between $L$ and $L^{\prime}$. Given a closed braid representation of $L$, we have

$$
\operatorname{sl}(L)=n_{+}-n_{-}-b,
$$

where $n_{+}\left(n_{-}\right)$is the number of positive (negative) crossings, and $b$ is the number of strings in the braid.

The stabilization of a transverse link represented as a braid is equivalent to the negative braid stabilization, i.e., adding an extra string and a negative kink to the braid. If $L_{\text {stab }}$ be the result of stabilization of $L$, then

$$
\operatorname{sl}\left(L_{\text {stab }}\right)=\operatorname{sl}(L)-2 .
$$

Note that the positive braid stabilization does not change the transverse type of the link.

We will often describe a braid by its braid word on the standard generators $\sigma_{1}, \sigma_{2}, \ldots$ and their inverses and draw associated braid diagrams. 


\section{Crossing resolution and contact surgery}

In this section, we establish the correspondence between the crossings of the braid diagram representing the transverse link $L$ and the surgeries required to obtain the contact manifold $\left(\Sigma(L), \xi_{L}\right)$ from $\left(S^{3}, \xi_{\text {std }}\right)$. This correspondence is our main tool: it allows to build contact surgery diagrams and plays the key role in other results of this paper.

3.1. Contact surgery. Let $K$ be a null-homologous Legendrian knot in a contact manifold $(Y, \xi)$. Legendrian surgery on $K$ is the surgery with coefficient $\operatorname{tb}(K)-1$; it is well known that a Legendrian surgery on $(Y, \xi)$ produces a new contact manifold $\left(Y^{\prime}, \xi^{\prime}\right)$, which is Stein fillable if $(Y, \xi)$ is. We often refer to Legendrian surgery as $(-1)$ contact surgery (comparing the surgery framing to the framing given by contact planes). It is also possible to make sense of $(+1)$ contact surgery (in fact, any rational $p / q$ surgery) [5]. The new contact manifold is obtained by cutting out a tubular neighborhood of the knot $K$ (i.e., a solid torus) and gluing it back in so that the contact structure on the solid torus matches the contact structure on its complement; when the surgery coefficient is $1 / q$ with $q \in \mathbb{Z}$, the result of this procedure is independent of choices. We refer the reader to $[\mathbf{5}, \mathbf{6}]$ for the details of this construction. We recall that $(+1)$ contact surgery is the operation inverse to the $(-1)$ contact (i.e., Legendrian) surgery, and note that the $(+1)$ surgery does not preserve Stein fillability or other similar properties of contact structures.

3.2. Surgery diagrams for double covers. We are now ready to relate the crossings of the braid diagram to contact surgeries. Roughly, a positive crossing gives a $(-1)$-surgery and a negative crossing a $(+1)$-surgery.

\section{Theorem 3.1.}

(1) If the transverse braid $L$ is obtained from the transverse braid $L_{+}$ by resolving a positive crossing, then $\left(\Sigma\left(L_{+}\right), \xi_{L_{+}}\right)$is obtained from $\left(\Sigma(L), \xi_{L}\right)$ by Legendrian surgery.

(2) If $L$ is obtained from $L_{-}$by resolving a negative crossing, then the contact manifold $\left(\Sigma\left(L_{-}\right), \xi_{L_{-}}\right)$is obtained from $\left(\Sigma(L), \xi_{L}\right)$ by $(+1)$ contact surgery.

Proof. We first consider a model example of two simple braids in $\left(S^{3}, \xi_{\text {std }}\right)$. Let $K \subset S^{3}$ be the transverse unknot given by the braid $\sigma_{1}$, and $K_{+}$be the transverse Hopf link given by $\sigma_{1}^{2}$. We claim that $\Sigma(K)=S^{3}$ and $\Sigma\left(K_{+}\right)=$ $L(2,1)=\mathbb{R} P^{2}$ with their (unique) tight contact structures. Indeed, since $K$ is the transverse unknot with $\operatorname{sl}(K)=-1$, it can be thought of as the binding of an open book decomposition of $S^{3}$ whose page is a disk. The branched double cover, then, is the same open book, giving the standard contact structure on $S^{3}$. The positive Hopf link $K_{+}$with $\operatorname{sl}\left(K_{+}\right)=0$ is the 

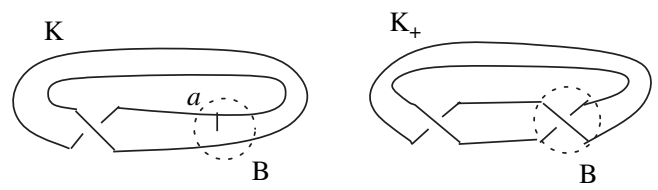

Figure 1. The branched double covers for the unknot and the Hopf link differ by regluing a solid torus which is a double cover of the ball $B$. This amounts to a surgery on the knot obtained as the branched double cover of the $\operatorname{arc} a$.

binding of an open book whose page is an annulus, and the monodromy is the positive Dehn twist. Doubling the monodromy, we see that $\Sigma\left(K_{+}\right)=L(2,1)$ and that the contact structure $\xi_{+}$is Stein fillable. (See [4] for the relation between open book decompositions and fillability.)

Now, $K$ and $K_{+}$agree in the complement of a ball $B$ containing one of the crossings of $K_{+}$(the boundary of this ball is shown in Figure 1 as a dotted circle). The double cover of this complement $S^{3} \backslash B$ branched along the two $\operatorname{arcs}$ of $K$ is a solid torus $M$. The ball $B$ contains two arcs of $K$ and two arcs of $K^{\prime}$, and the branched double covers $\Sigma(K)$ and $\Sigma\left(K_{+}\right)$are both obtained from $M$ by attaching another solid torus $N$, a double cover of $B$ branched along two arcs. This means that $\Sigma\left(K_{+}\right)$is obtained as a surgery on a knot in $\Sigma(K)$. This knot is the branched double cover $\tilde{a}$ of the arc $a$ connecting the two strands of $K$ inside $B$; it represents the longitude of the solid torus $N$. Therefore, we are doing surgery on an unknot in $S^{3}$; since the result of this surgery is $L(2,1)$, the surgery coefficient is -2 . (For surgeries relating branched double covers, the surgery coefficient is always integral.)

Our goal is to put this surgery into a contact context. First, we can assume that the $\operatorname{arc} a$ is Legendrian (and so is $\tilde{a}$ ). The surgery on the contact $S^{3}$ then becomes contact surgery on a Legendrian unknot; if $\operatorname{tb}(\tilde{a})=-n$ for some $n \geq 1$, then we must be doing $(n-2)$-contact surgery (the result of this surgery is not unique unless $n-2= \pm 1$ ). Our goal is to show that $n=1$, so that we have the Legendrian surgery on the standard Legendrian unknot in $S^{3}$. To this end, we will show that the resulting contact structure is overtwisted whenever $n>1$. Indeed, consider $(+1)$-contact surgery on
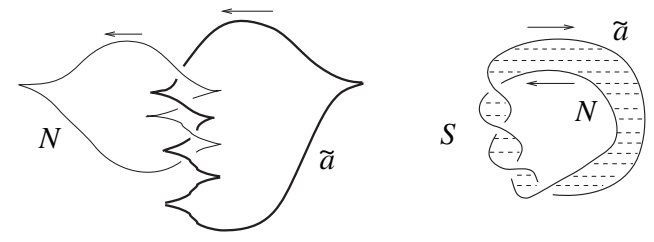

Figure 2. The unknot $N$ bounds an overtwisted disk in the surgered manifold; part of the disk is formed by the surface $S$. 
an unknot with $t b=-3$ (Figure 2 ). The surface framing of the unknot $\tilde{a}$ given by the Seifert surface $S$ of the link $N \cup \tilde{a}$ in this figure equals -2 , which is the Dehn surgery coefficient. Then, the new meridional disk glued together with $S$ gives an embedded disk $D_{0}$ bounded by $N$ in the surgered manifold. The Thurston-Bennequin number of $N$ is -2 , which is the same as the surface framing of $N$ determined by the disk $D_{0}$. It follows that $D_{0}$ is an overtwisted disk. (A similar argument for an overtwisted sphere is given in [6]). There are two more unknots with tb $=-3$, one with three kinks on the right and one on the left, and the other with two kinks on each side; they can be treated in the same way (putting $N$ on the left or on the right and orienting the link as needed).

If $\operatorname{tb}(\tilde{a})=-n<-3$, the $(n-2)$-contact surgery is no longer uniquely defined, but the same argument applies, and we can always find an unknot $N$ that bounds an overtwisted disk in the surgered manifold. One possible picture for $\tilde{a}$ and $N$ is shown in Figure 3 (note that there are a few choices for the contact surgery even when $\tilde{a}$ is fixed, see $[\mathbf{6}])$. The remaining case is $\operatorname{tb}(\tilde{a})=-2$. Then, the surgery coefficient matches the Thurston-Bennequin number (i.e., we are attempting to do 0-contact surgery); in this case, our Legendrian unknot bounds an overtwisted disk in the surgered manifold.

Therefore, we see that the contact manifold $\left(\Sigma\left(K_{+}\right), \xi_{+}\right)$is the result of the Legendrian surgery on the unknot in $(\Sigma(K), \xi)$ with tb $=-1$. This unknot is a Legendrian representative of the lifting of a chord $a$ of $K$, shown in Figure 1. Because $(+1)$-contact surgery and Legendrian surgery are inverse to one another, we can also say that $(\Sigma(K), \xi)$ is obtained from $\left(\Sigma\left(K_{+}\right), \xi_{+}\right)$by the contact $(+1)$-surgery on $\tilde{a}$.

We are now ready to prove part (1) of the theorem. We know that the contact manifold $\left(\Sigma\left(L_{+}\right), \xi_{L_{+}}\right)$is obtained by some contact surgery on the Legendrian knot $\tilde{a}$ obtained as the branched double cover of an arc connecting two strings of the braid $L$. The framing of this surgery is a purely local question, so from our model example, we see that it must be the Legendrian framing.

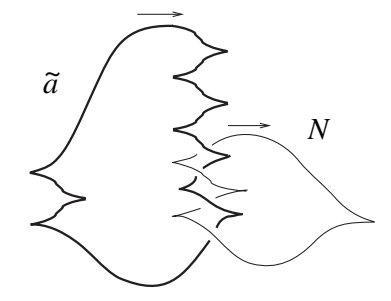

Figure 3. As in the previous picture, the unknot $N$ bounds an overtwisted disk in the surgered manifold. 
Part (2) also follows from the local model: indeed, the link $K_{+}$can be obtained from $K$ by resolving a negative crossing (we first change the diagram by a Reidemeister move to introduce two new crossings, a positive and a negative one, that cancel each other).

We can now give a contact surgery diagram for a double cover branched over an arbitrary transverse braid $L$ on $n$ strings. Inserting the factors $\sigma_{i} \sigma_{i}^{-1}$ if necessary, we may assume that the braid word contains each $\sigma_{i}$ with $i=1, \ldots, n$. Therefore, the braid $L$ can be obtained from the braid $U=\sigma_{1} \sigma_{2} \cdots \sigma_{n}$ by introducing some extra (positive and negative) crossings. Obviously, the transverse link $U$ is the unknot with $\operatorname{sl}(U)=-1$, and so the double cover of $S^{3}$ branched over $U$ is $\left(S^{3}, \xi_{\text {std }}\right)$. Now, $\left(\Sigma(L), \xi_{L}\right)$ is the result of a contact surgery on a link in the standard sphere: each "extra" crossing of $L$ gives a $(-1)$ or a $(+1)$ contact surgery on a Legendrian unknot (depending on the sign of the crossing). These Legendrian unknots are the components for the surgery link for $\left(\Sigma(L), \xi_{L}\right)$; it remains to understand how they are linked. As explained in [11, Lemma 3.6], the linking number between two components is given by the number of twists in the unknot between the attaching points of the two chords. In our case, because of the special position of the unknot $U$ and the chords, this linking number is always zero or \pm 1 and can be easily determined by untwisting the unknot (we can also pinpoint the sign of the linking number if we orient the surgery link by using the blackboard framing of $U$, as described in [11]). It is convenient to picture $U$ as a round circle and to mark on it the attaching points for the chords given by the crossings.

Example 3.2. Two examples are shown in Figure 4: the double branched cover for the right-handed trefoil (the braid $\sigma_{1}^{3}$ ) gives the (unique) Stein fillable structure on $-L(3,1)$, and the double cover branched over the transverse unknot with $\mathrm{sl}=-3$ is an overtwisted sphere. For the surgery diagrams here and later on, we choose the more familiar contact form $\xi_{\text {std }}=\operatorname{ker}(d z-x d y)$, which is isotopic, but not identical to the rotationally

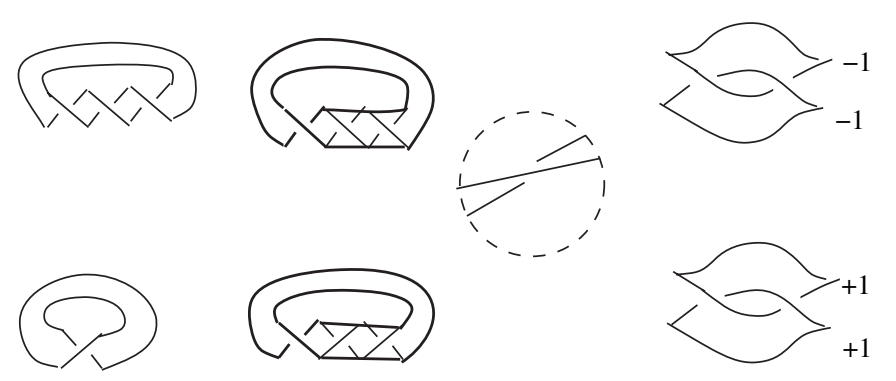

Figure 4. Constructing surgery diagrams: the trefoil and the unknot. 
symmetric contact form used for the braid representation of the transverse links. We hope that this will lead to no confusion.

Remark 3.3. It is not necessary to single out the braid word that gives the unknot: we can as well start from the trivial braid and obtain $\left(\Sigma(L), \xi_{L}\right)$ as a result of surgery on $\left(\# S^{1} \times S^{2}, \xi_{0}\right)$ (where $\xi_{0}$ is the unique Stein fillable contact structure). However, we find surgeries on the sphere more practical, especially for the next subsection.

3.3. Birman-Menasco braids. We can now use the strategy from the previous subsection to construct surgery diagrams for the Birman-Menasco braids. We make Theorem 1.1 more precise:

Theorem 3.4. Let $p, q, r>1$ be integers with $p+1 \neq q \neq r$ and consider the transverse braids

$$
K_{1}=\sigma_{1}^{2 p+1} \sigma_{2}^{2 q} \sigma_{1}^{2 r} \sigma_{2}^{-1} \quad \text { and } \quad K_{2}=\sigma_{1}^{2 p+1} \sigma_{2}^{-1} \sigma_{1}^{2 r} \sigma_{2}^{2 q} .
$$

The branched double covers $\left(\Sigma\left(K_{1}\right), \xi_{K_{1}}\right)$ and $\left(\Sigma\left(K_{2}\right), \xi_{K_{2}}\right)$ are contactomorphic: indeed, they can be described by the same contact surgery diagram.

Remark 3.5. As mentioned in the introduction, $K_{1}$ and $K_{2}$ have the same self-linking number and are isotopic as smooth knots, but not as transverse knots.

Proof. The two braids together with the surgery diagram for both double covers are shown in Figure 5. (The circles with the attaching chords help understand where the diagram comes from.)

Remark 3.6. We can also study the manifold $\left(\Sigma(L), \xi_{L}\right)$ in terms of its open book decomposition. Indeed, the page of such open book is given by a branched double cover of a disk which is transverse to all strings of the braid. The monodromy comes from the braid monodromy: each generator $\sigma_{i}$ corresponds to a Dehn twist. The manifold $\left(\Sigma(L), \xi_{L}\right)$ can then be exhibited as the boundary of an achiral Lefschetz fibration. The open book description, together with the results of [4], would lead to an alternative quick proofs for the next section. The surgery diagrams are more convenient for our purposes because they fit nicely with the arguments of [11].

\section{Quasipositive braids and stabilizations}

To prove Propositions 1.3 and 1.4, we turn our attention to transverse links represented as quasipositive braids, as well as to those which can be obtained as transverse stabilizations.

Recall [17] that a braid is called quasipositive if its braid word is a product of conjugates of the form $w \sigma_{i} w^{-1}$, where $w$ is an arbitrary element of the braid group. 


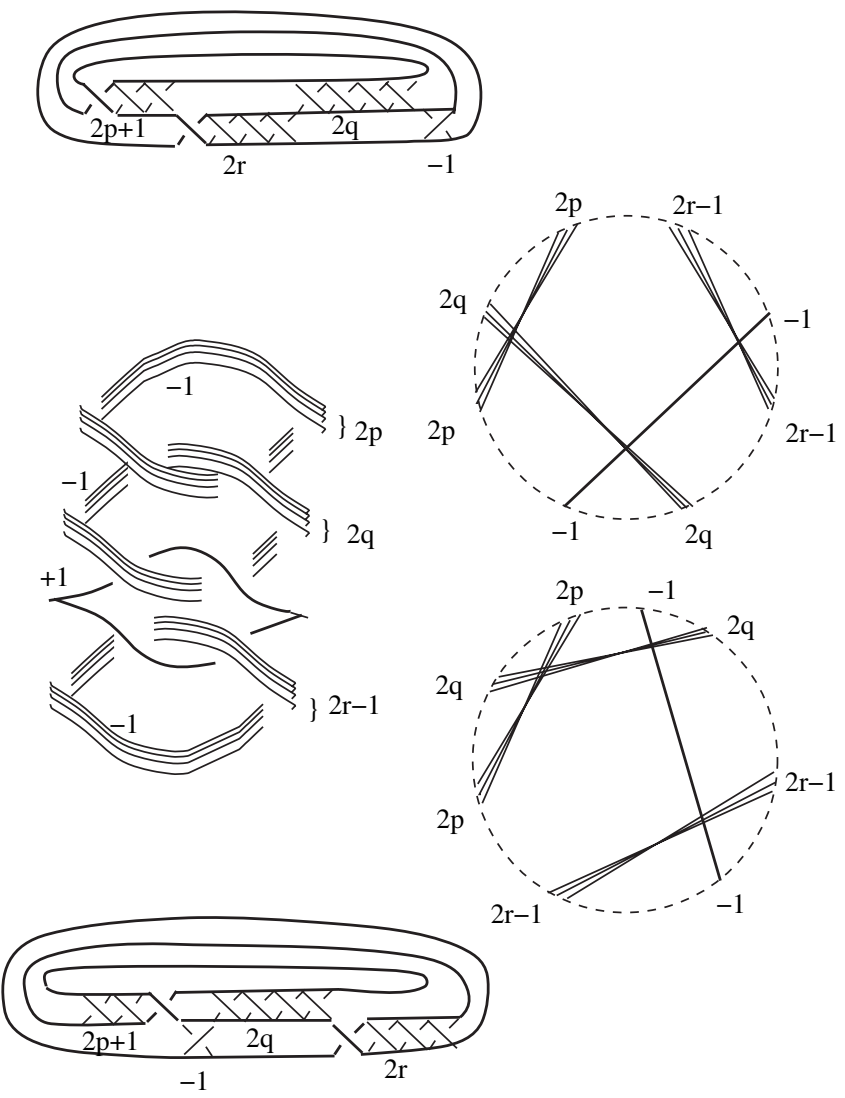

Figure 5. Double covers are contactomorphic.

Proof of Proposition 1.3. Resolving a few positive crossings, we convert the braid representing $L$ into a braid equivalent to a trivial one (of the same braid index). For the trivial braid $\mathcal{O}$, the contact manifold $\left(\Sigma(\mathcal{O}), \xi_{\mathcal{O}}\right)$ is a connected sum of several copies of $\left(S^{1} \times S^{2}, \xi_{0}\right)$, which is Stein fillable. By Theorem 3.1, $\left(\Sigma(L), \xi_{L}\right)$ is obtained from $\left(\Sigma(\mathcal{O}), \xi_{\mathcal{O}}\right)$ by a sequence of Legendrian surgeries, so it will be Stein fillable, too.

Conversely, a stabilized transverse link gives some $(+1)$ surgeries in the contact surgery diagram for the double branched cover.

Proof of Proposition 1.4. Suppose that a transverse link $L_{\text {stab }}$ is obtained as a transverse stabilization of a link $L$. Then $L_{\text {stab }}$ can be represented as a braid with a negative kink. Now use the algorithm from Section 3 to translate the braid representation into a contact surgery diagram. We see that the diagram breaks into two pieces: two $(+1)$ surgeries on two linked Legendrian unknots (cf. Example 3.2, Figure 4), isolated from everything 
else, and the surgery diagram for $\left(\Sigma(L), \xi_{L}\right)$. It follows that $\left(\Sigma\left(L_{\text {stab }}\right), \xi_{\text {stab }}\right)$ is the connected sum of $\left(\Sigma(L), \xi_{L}\right)$ and an overtwisted $S^{3}$, so it is overtwisted.

Corollary 4.1. Let $T$ be a transverse link smoothly isotopic to a $(p, q)$ torus link, $p, q>0$. If $\operatorname{sl}(T)=\mathrm{sl}_{\max }=p q-p-q$, then $\left(\Sigma(T), \xi_{T}\right)$ is Stein fillable. Otherwise $\left(\Sigma(T), \xi_{T}\right)$ is overtwisted.

Proof. We make use of the transverse simplicity of torus links [18], that is, the fact that the transverse $(p, q)$ torus link is uniquely determined by its self-linking number. When $\operatorname{sl}(T)=p q-p-q$, the link $T$ can be represented as the obvious positive braid, so the branched double cover is Stein fillable. For smaller values of sl, the transverse link $T$ is obtained as a result of a few stabilizations of that braid, which proves overtwistedness.

\section{5. $\operatorname{Spin}^{c}$ structures and the three-dimensional invariant}

A contact structure $\xi$ on a contact manifold $Y$ induces a $\operatorname{Spin}^{c}$ structure $\mathfrak{s}$ on $Y$. When $c_{1}(\mathfrak{s})$ is torsion, there is also the three-dimensional invariant $d_{3}(\xi)[\mathbf{7}]$. If $(Y, \xi)$ is the boundary of an almost-complex 4-manifold $(X, J)$, this invariant is given by

$$
d_{3}(\xi)=\frac{1}{4}\left(c_{1}^{2}(J)-2 \chi(X)-3 \operatorname{sign}(X)\right) .
$$

As described in Section 3, given a braid presentation for a transverse knot $K$, we can find a contact surgery description of $\left(\Sigma(K), \xi_{K}\right)$. More precisely, assuming that the braid has $b$ strings, and the braid word contains each $\sigma_{i}$ for $i=1, \ldots, b$ and has $l+b$ positive entries and $m$ negative entries, we see that $\left(\Sigma(K), \xi_{K}\right)$ is obtained from $\left(S^{3}, \xi_{\text {std }}\right)$ by a sequence of $l$ Legendrian surgeries and $m(+1)$ contact surgeries, so that all the surgeries are performed on the standard Legendrian unknot.

The results of $[6]$ can be used to understand the $\operatorname{Spin}^{c}$ structure and the $d_{3}$ invariant for the contact manifold $\left(\Sigma(L), \xi_{L}\right)$ for a transverse link $L$.

Let $X$ be the 4-manifold obtained from $D^{4}$ by attaching the 2-handles as dictated by the $( \pm 1)$-surgery diagram. (If the link $L$ has no negative crossings, $X$ is Stein.) Following $[6]$, consider an almost-complex structure $J$ defined on $X$ in the complement of $m$ balls lying in the interior of the $(+1)$-handles of $X$. As shown in $[6], J$ induces a $\operatorname{Spin}^{c}$ structure $s_{J}$ which extends to all of $X$. The $d_{3}$ invariant of $\xi_{L}$ can be computed as

$$
d_{3}\left(\xi_{K}\right)=\frac{1}{4}\left(c_{1}^{2}\left(\mathfrak{s}_{J}\right)-2 \chi(X)-3 \operatorname{sign}(X)\right)+m .
$$

This formula is very similar to the case where $(X, J)$ is almost-complex, except that there is a correction term of +1 for each $(+1)$-surgery.

Now, suppose that a 2-handle is attached to the 4-manifold $X$ in the process of Legendrian surgery on a knot $K$, and denote by $[S]$ the homology 
class that arises from the Seifert surface of $K$ capped off inside the handle. It is well known [7] that $c_{1}\left(\mathfrak{s}_{J}\right)$ evaluates on $[S]$ as the rotation number of the Legendrian knot $K$. Furthermore, it is shown in [6] that the same result is true for $(+1)$-contact surgeries (for the $\operatorname{Spin}^{c}$ structure $s_{J}$ on $X$ described above). Thus, we see that in our case $c_{1}\left(\mathfrak{s}_{J}\right)$ evaluates as 0 on each homology generator corresponding to either a $(-1)$ or a $(+1)$ surgery, because the rotation number of the standard Legendrian unknot is 0 . We conclude that $c_{1}\left(\mathfrak{s}_{J}\right)=0$. Since $\mathfrak{s}_{\xi}$ is the restriction of $\mathfrak{s}_{J}$, we have

Lemma 5.1. For any transverse link $L, c_{1}\left(\mathfrak{s}_{\xi_{L}}\right)=0$.

The Euler characteristic of the manifold $X$ is $1+\#(2$-handles), which is $1+l+m$; formula (5.1) simplifies as $d_{3}\left(\xi_{L}\right)=-\frac{3}{4} \sigma(X)-\frac{1}{2}(l-m)-\frac{1}{2}=$ $-\frac{3}{4} \sigma(X)-\frac{1}{2} \operatorname{sl}(L)-\frac{1}{2}$.

Our next task is show that this expression depends on the topological type of $L$ and $\operatorname{sl}(L)$ only.

Lemma 5.2. Suppose that two closed braids $L$ and $L^{\prime}$ are isotopic as smooth knots and that $\operatorname{sl}(L)=\operatorname{sl}\left(L^{\prime}\right)$. Then $d_{3}\left(\xi_{L}\right)=d_{3}\left(\xi_{L^{\prime}}\right)$.

Proof. Since the braids $L$ and $L^{\prime}$ give rise to isotopic knots, by the classical Markov theorem [19] $L^{\prime}$ can be obtained from $L$ by a sequence of braid isotopies and (positive and negative) braid stabilizations and destabilizations. Braid isotopies do not change the transverse link type, and neither do positive stabilizations; this means that both the self-linking number and the $d_{3}$ invariant remain unchanged. A negative stabilization changes both $\operatorname{sl}(L)$ and $d_{3}\left(\xi_{L}\right)$, but it is easy to keep track of the changes. Indeed, the selflinking number decreases by 2 . The branched double cover $\left(\Sigma\left(L_{\text {stab }}\right), \xi_{L_{\text {stab }}}\right)$ is obtained from $\left(\Sigma(L), \xi_{L}\right)$ by the connected sum with an overtwisted sphere which is a $(+1)$ contact surgery on two linked Legendrian unknots (cf. Proposition 1.4). It follows from (5.1) that the $d_{3}$ invariant increases by 1. Since $\operatorname{sl}(L)=\operatorname{sl}\left(L^{\prime}\right)$, every negative stabilization must be compensated by a destabilization, so that we have the same number of stabilizations and destabilizations. Then, we must have $d_{3}\left(\xi_{L}\right)=d_{3}\left(\xi_{L^{\prime}}\right)$.

\section{Ozsváth-Szabó invariants}

In this section, we study the Ozsváth-Szabó invariants of the contact structures on the branched double covers. As the author learned upon completion of this paper, the same question was independently studied by John Etnyre, who obtained similar results.

6.1. A brief review. We quickly recall a few facts about the Heegaard Floer homology groups here, referring the reader to the papers of Ozsváth and Szabó for details. We use coefficients in $\mathbb{Z}$. Given a 3-manifold $Y$ equipped with a Spin ${ }^{c}$ structure $\mathfrak{s}$, Ozsváth and Szabó use a Floer-theoretic 
construction to define a homology group $\widehat{\mathrm{HF}}(Y, \mathfrak{s})$. Together, these groups form $\widehat{\mathrm{HF}}(Y)=\bigoplus_{\mathfrak{s} \in \operatorname{Spin}^{c}(Y)} \widehat{\mathrm{HF}}(Y, \mathfrak{s})$. Cobordisms induce a map on Floer homology. More precisely, a $\operatorname{Spin}^{c} \operatorname{cobordism~}(W, \mathfrak{s})$ gives a map

$$
F_{W, \mathfrak{s}}: \widehat{\mathrm{HF}}\left(Y_{1}, \mathfrak{s} \mid Y_{1}\right) \longrightarrow \widehat{\mathrm{HF}}\left(Y_{2}, \mathfrak{s} \mid Y_{2}\right) .
$$

The map $F_{W}: \widehat{\mathrm{HF}}\left(Y_{1}\right) \rightarrow \widehat{\mathrm{HF}}\left(Y_{2}\right)$ is defined by summing over all $\operatorname{Spin}^{c}$ structures.

For a manifold $Y$ equipped with a contact structure $\xi$, Ozsváth and Szabó [9] define a contact invariant $c(\xi) \in \widehat{\mathrm{HF}}(-Y)$. The element $c(\xi)$ is welldefined up to a sign and lives in the $\operatorname{Spin}^{c}$ component $\widehat{\mathrm{HF}}\left(-Y, \mathfrak{s}_{\xi}\right)$ associated to the contact structure. Cobordisms given by Legendrian surgeries respect $c(\xi)$.

Proposition 6.1 [20]. Let $\left(Y^{\prime}, \xi^{\prime}\right)$ be obtained from $(Y, \xi)$ by a Legendrian surgery. Denote by $W$ the surgery cobordism, and let $F_{W}: \widehat{\mathrm{HF}}\left(-Y^{\prime}\right) \rightarrow$ $\widehat{\mathrm{HF}}(-Y)$ be the associated map. Then $F_{W}\left(c\left(\xi^{\prime}\right)\right)=c(\xi)$.

When $\mathfrak{s}$ is a torsion $\operatorname{Spin}^{c}$ structure, $\widehat{\mathrm{HF}}(Y)$ is a $\mathbb{Z}$-graded group. (Strictly speaking, this grading gr takes values in $\mathbb{Q}$; it is a $\mathbb{Z}$-grading shifted by a rational constant.) The degree of $c(\xi)$ is closely related to the threedimensional invariant of $\xi$.

Proposition 6.2 [9]. Suppose $\mathfrak{s}_{\xi}$ is torsion. Then $c(\xi)$ is a homogeneous element of degree $\operatorname{gr}(c(\xi))=d_{3}(\xi)+\frac{1}{2}$.

Finally, we have the following important fact.

Theorem $6.3[9]$.

(1) If the contact structure $\xi$ is overtwisted, then $c(\xi)=0$.

(2) If $\xi$ is Stein fillable, then $c(\xi) \neq 0$. Indeed, $c(\xi) \in \widehat{\mathrm{HF}}(-Y)$ is a primitive element.

6.2. Contact invariants of double covers. Combining the results of the previous sections with the properties of the Heegaard Floer contact invariant, we immediately get the following propositions.

Proposition 6.4. Suppose that the transverse link $L$ is the result of transverse stabilization of another link. Then $c\left(\xi_{L}\right)=0$.

Proposition 6.5. Let the transverse link $L$ be represented as a quasipositive braid. Then $c\left(\xi_{L}\right) \neq 0$. Indeed, it is a homogeneous primitive element in $\widehat{\mathrm{HF}}(-\Sigma(L))$, whose grading is given by

$$
\operatorname{gr}\left(\xi_{L}\right)=-\frac{3}{4} \sigma(X)-\frac{1}{2} \operatorname{sl}(L)
$$

where $X$ is the 4-manifold described in Section 5. The latter expression depends on the topological type of $L$ and its self-linking number only. 
Proposition 6.6. Let the link $L$ be obtained from the link $L_{+}$by resolving a positive crossing. Let $W$ be the associated surgery cobordism between the two branched double covers, and $F_{W}: \widehat{\mathrm{HF}}\left(-\Sigma\left(L_{+}\right)\right) \rightarrow \widehat{\mathrm{HF}}(-\Sigma(L))$ the induced map on homology. Then $F_{W}\left(c\left(\xi_{L_{+}}\right)\right)=c\left(\xi_{L}\right)$.

We also have

Proposition 6.7. For the connected sum $L_{1} \# L_{2}$ of transverse links $L_{1}$ and $L_{2}, c\left(\xi_{L_{1} \# L_{2}}\right)=c\left(\xi_{L_{1}}\right) \otimes c\left(\xi_{L_{2}}\right)$.

Proof. The contact manifold $\left(\Sigma\left(L_{1} \# L_{2}\right), \xi_{L_{1} \# L_{2}}\right)$ is the connected sum of $\left(\Sigma\left(L_{1}\right), \xi_{L_{1}}\right)$ and $\left(\Sigma\left(L_{2}\right), \xi_{L_{2}}\right)$, so the proposition follows from the connected sum statement in $[\mathbf{9}]$.

The following proposition is useful in calculations and shows that $c\left(\xi_{L}\right)=$ 0 in many cases.

Proposition 6.8. Suppose that the transverse link $L$ is represented by a closed braid such that its braid word contains a factor of $\sigma_{i}^{-1}$ but no $\sigma_{i}$ 's for some $i>0$. (This means that all the crossings in the braid diagram on the level between $(i-1)$-th and $i$-th string are negative.) Then $c\left(\xi_{L}\right)=0$.

Proof. First of all, we delete all $\sigma_{i}^{-1}$ but one from the braid word, obtaining a link that decomposes as a connected sum of two links (connected by a negative crossing, the $\sigma_{i}^{-1}$ that remains). Deleting further negative crossings and inserting new positive crossings into both components of the connected sum, we obtain a link $L^{\prime}$ given by two positive torus knots connected by a negative crossing. Topologically, the link $L^{\prime}$ is just the connected sum of two torus knots; as a transverse link, it does not have maximal self-linking number (because we can connect the two components by a positive crossing instead of a negative one to increase sl). Connected sums of torus knots are transversely simple [21], so $L^{\prime}$ is the transverse stabilization of another link. By Proposition 1.4, $c\left(\xi_{L^{\prime}}\right)=0$. Repeated use of Proposition 6.6 now implies that $c\left(\xi_{L}\right)=0$.

Corollary 6.9. Let $L$ be a transverse representative of a negative torus link. Then $c\left(\xi_{L}\right)=0$.

\section{Relation with Khovanov homology}

Now we explore the connection to the Khovanov homology mentioned in the introduction. We consider the Khovanov homology with $\mathbb{Z} / 2 \mathbb{Z}$ coefficients.

We very briefly recall the relevant constructions. (Our review here is similar to the one in $[\mathbf{2 2}]$.) The starting point for defining the Khovanov homology [23] of a smooth link $L \subset S^{3}$ is a link diagram (which we still denote $L$ ). Let $n$ be the number of crossings in $L$. Khovanov homology $\mathrm{Kh}(L)$ is the homology of the chain complex $C \mathrm{Kh}(L)$, which is formed by 


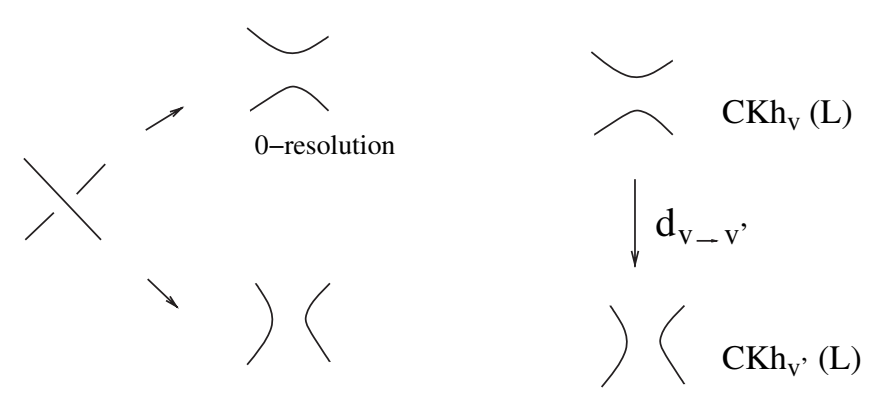

1-resolution

Figure 6. Crossing resolutions and differentials.

considering all "complete resolutions" of $L$. Each crossing can be resolved in two ways, ("0-resolution" and "1-resolution", see Figure 6), so that complete resolutions can be written as $L_{v}$, indexed by $v \in[0,1]^{n}$. Each complete resolution consists of a few disjoint circles; let $L_{v}$ consist of $m$ circles. To obtain the generators for $C \operatorname{Kh}\left(L_{v}\right)$, label each of the circles by either $\mathbf{u}_{-}$or $\mathbf{u}_{+}$(that is, set $C \operatorname{Kh}\left(L_{v}\right)=U^{\otimes m}$, where $U$ is a vector space with basis $\left.\left\{\mathbf{u}_{-}, \mathbf{u}_{+}\right\}\right)$. The underlying space for the Khovanov complex is then $C \operatorname{Kh}(L)=\oplus_{v \in\{0,1\}^{n}} C \operatorname{Kh}\left(L_{v}\right)$. The differential $d$ is defined as a sum of its components $d_{v \rightarrow v^{\prime}}: C \mathrm{Kh}(v) \rightarrow C \mathrm{Kh}\left(v^{\prime}\right)$ for all $v, v^{\prime}$ which are adjacent vertices of the cube $[0,1]^{n}$, so that $L_{v}^{\prime}$ can be obtained from $L_{v}$ by changing a 0 -resolution of one crossing into a 1-resolution. The chain complex $C \operatorname{Kh}(L)$ is bi-graded; up to a correction term, the homological grading of all elements in $C \mathrm{Kh}\left(L_{v}\right)$ is given by the number of zeroes among the coordinates of $v \in\{0,1\}^{n}$, so that $d$ raises the homological grading by 1 . We do not describe the differential in detail, nor do we discuss the quantum grading on $C \operatorname{Kh}(L)$. (The reader is referred to the original paper [23] or to surveys such as $[\mathbf{1 2}]$.)

The reduced complex $\widetilde{C K h}(L)$ is defined for a link with a marked point. Each complete resolution now has one marked circle; let $C \mathrm{Kh}_{\mathbf{u}_{-}}(L)$ be generated by those $\mathbf{u}_{ \pm} \otimes \cdots \otimes \mathbf{u}_{ \pm}$that have the label $\mathbf{u}_{-}$on the marked circle. Then, the reduced complex is defined as $\widetilde{C K h}(L)=C \operatorname{Kh}(L) / C \operatorname{Kh}_{\mathbf{u}_{-}}(L)$. The group $\widetilde{\mathrm{Kh}}(L)$ is the reduced Khovanov homology group.

Given a transverse link $L \subset S^{3}$, we define an invariant $\psi(L) \in \widetilde{\mathrm{Kh}}(L)$ [10]. We represent $L$ as a transverse braid and take the oriented resolution $L_{o}$ of the braid diagram (i.e., the resolution that consists of parallel strings). For unreduced Khovanov homology, we pick the element $\mathbf{u}_{-} \otimes \mathbf{u}_{-} \otimes \cdots \otimes$ $\mathbf{u}_{-} \in C \operatorname{Kh}(L)$, that is, we label every component of the oriented resolution with a $\mathbf{u}_{-}$. For reduced homology, there is an isomorphism $\widetilde{C K h}(L) \cong$ $C \mathrm{Kh}_{\mathbf{u}_{-}}(L)$ obtained by replacing the label $\mathbf{u}_{+}$on the marked component 
with a $\mathbf{u}_{-}$; we define $\tilde{\psi}(L)$ to be the element of $\widetilde{C \mathrm{Kh}}(L)$ corresponding to $\mathbf{u}_{-} \otimes \cdots \otimes \mathbf{u}_{-} \in C \mathrm{Kh}_{\mathbf{u}_{-}}(L)$ under this isomorphism. We show that $\tilde{\psi}(L)$ descends to $\psi(L) \in \widetilde{\mathrm{Kh}}(L)$ and gives an invariant of the transverse link, well-defined up to a sign. The properties of $\psi(L)$ are similar to those of $c\left(\xi_{L}\right)$; in fact, we prove analogs of results of Section 6 . In the introduction, we have conjectured that $\psi(L)$ "corresponds" to $c\left(\xi_{L}\right)$ under the spectral sequence of $[\mathbf{1 1}]$ which relates $\widetilde{\mathrm{Kh}}(L)$ and $\widehat{\mathrm{HF}}(-\Sigma(L))$.

To be more precise, we consider the special case when the link $L$ is alternating; in this case, the spectral sequence collapses, giving an isomorphism between $\widetilde{\mathrm{Kh}}(L)$ and $\widehat{\mathrm{HF}}(-\Sigma(L))$. We recall how this isomorphism is established, describing $\widehat{\mathrm{HF}}(-\Sigma(L))$ as the homology of the following filtered chain complex [11]. Given a link diagram $L$, consider again all complete resolutions $L_{v}, v \in\{0,1\}^{n}$. For each $L_{v}$, let $Y_{v}=\Sigma\left(L_{v}\right)$ be the double cover of $S^{3}$ branched over $L_{v}$. (Since $L_{v}$ is simply the disjoint union of say $m+1$ circles, $Y_{v}=\#_{m} S^{1} \times S^{2}$.) Now, let $E^{1}=\oplus_{v \in\{0,1\}^{n}} \widehat{\mathrm{HF}}\left(-Y_{v}\right)$ be the underlying space of the chain complex, and construct the differential $D=D^{1}$ as follows. As in Khovanov's theory, $D$ is the sum of its components $D_{v \rightarrow v^{\prime}}: \widehat{\mathrm{HF}}\left(-Y_{v}\right) \rightarrow \widehat{\mathrm{HF}}\left(-Y_{v^{\prime}}\right)$ for all adjacent $v, v^{\prime}$ (such that $L_{v}^{\prime}$ is obtained from $L_{v}$ by changing a 0-resolution of one crossing into a 1-resolution). Then, $Y_{v}$ is obtained from $Y_{v^{\prime}}$ by a single 2-handle attachment. The map $D_{v \rightarrow v^{\prime}}$ is then defined as a map on Heegaard Floer homology associated to the handle attachment cobordism. The filtration grading on $E^{1}$ parallels the homological grading in Khovanov's theory; again, on $\widehat{\mathrm{HF}}\left(-Y_{v}\right)$, it is given by the number of zeroes among the coordinates of $v \in\{0,1\}^{n}$ (it is convenient to introduce a correction term, too, so that the two gradings are the same).

Theorem 7.1 [11]. Let $L$ be an alternating link, and fix its alternating diagram. The homology of the filtered chain complex $\left(E^{1}, D\right)$ is $\widehat{\mathrm{HF}}(-\Sigma(L))$. On the other hand, the associated graded complex of $\left(E^{1}, D\right)$ is isomorphic to $(\widetilde{C \mathrm{Kh}}(L), d)$. (For both theories, the coefficients are taken in $\mathbb{Z} / 2 \mathbb{Z}$.)

The isomorphism between the two chain complexes comes as part of the construction: indeed, $\widehat{\mathrm{HF}}\left(-Y_{v}\right)=\widehat{\mathrm{HF}}\left(\#_{m} S^{1} \times S^{2}\right)=\widetilde{C \mathrm{Kh}}\left(L_{v}\right)$, and the maps $D_{v \rightarrow v^{\prime}}$ are the same as $d_{v \rightarrow v^{\prime}}$ under this equivalence. When the link diagram is fixed, this provides a canonical isomorphism between $\widetilde{\mathrm{Kh}}(L)$ and the associated graded group of $\widehat{\mathrm{HF}}(-\Sigma(L))$. (The latter is non-canonically isomorphic to $\widehat{\mathrm{HF}}(-\Sigma(L))$.)

Remark 7.2. As notation suggests, $\left(E^{1}, D^{1}\right)$ is the first term of a certain spectral sequence [11]. This spectral sequence has "higher order" differentials defined via maps $D_{v \rightarrow v^{\prime}}$, with $v$, $v^{\prime}$ not necessarily adjacent. For a general smooth link, its $E^{2}$ term gives $\widetilde{\mathrm{Kh}}(L)$, and $E^{\infty}=\widehat{\mathrm{HF}}(-\Sigma(L))$. 
One of the key features of the Heegaard Floer theory is the surgery exact triangle. In the correspondence of [11], it parallels the skein exact sequence for the Khovanov homology. The skein exact sequence for a link $L$ relates $\widetilde{\mathrm{Kh}}(L), \widetilde{\mathrm{Kh}}\left(L_{0}\right)$, and $\widetilde{\mathrm{Kh}}\left(L_{1}\right)$, where $L_{0}$ and $L_{1}$ stand for the 0and 1-resolution of a given crossing of $L$. The surgery exact sequence for a 3-manifold $Y$ and a framed knot $\gamma \in Y$ relates $\widehat{\mathrm{HF}}(Y), \widehat{\mathrm{HF}}\left(Y_{1}(\gamma)\right)$, and $\widehat{\mathrm{HF}}\left(Y_{0}(\gamma)\right)$, where the manifolds $Y_{1}(\gamma)$ and $Y_{0}(\gamma)$ are obtained as the result of 1- resp. 0-surgery on $K$. Since crossing resolutions for links induce surgeries on the branched double covers, the surgery triangle relates the Heegaard Floer homology groups of manifolds $\Sigma(L), \Sigma\left(L_{0}\right)$, and $\Sigma\left(L_{1}\right)$, and the resulting exact sequence looks very similar to the skein sequence for the Khovanov homology. When the link $L$ is alternating (and so are its resolutions $L_{0}$ and $\left.L_{1}\right)$, the two exact sequences fit together nicely.

Lemma 7.3. Let the link $L$ be given by an alternating diagram, and let the links $L_{0}$ and $L_{1}$ be obtained by the 0 - and 1-resolution of a given crossing. Then the diagram

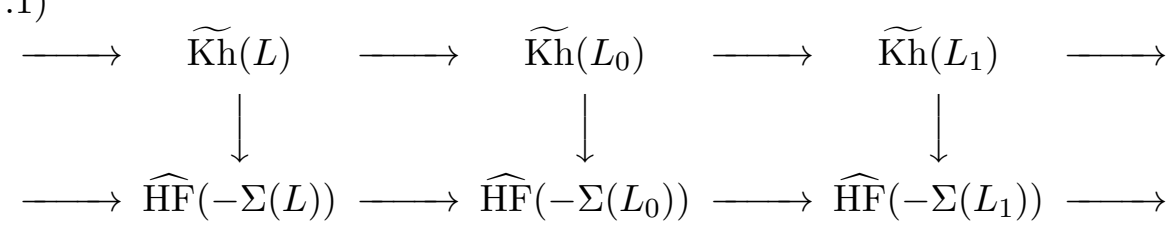

commutes. (That is, the two squares shown commute, and the third square implicit in the diagram also commutes.) Here the maps on the Heegaard Floer homology are induced by handle attachments, the maps on the Khovanov homology are induced by the crossing change, and the vertical maps are the isomorphisms provided by Theorem 7.1; to simplify notation here and below, we write $\widehat{\mathrm{HF}}(-\Sigma(L))$ for the corresponding associated graded group.

As Peter Ozsváth explained to the author, the proof of this lemma follows from the arguments and techniques of [11]. (In fact, we should consider maps on the corresponding filtered and associated graded complexes.) To avoid a lengthy review, we do not include this proof here. Instead, we consider a simple example which illustrates the commutativity of the diagram (7.1) and the interplay between $\psi(L)$ and $c\left(\xi_{L}\right)$.

Example 7.4. Consider the transverse unknots $L^{+}$and $L^{-}$(Figure 7 ), given by braid diagrams with one positive resp. one negative crossing, and their 0- and 1-resolutions. The exact triangles in the Heegaard Floer and the Khovanov theory look as follows. For the link $L^{+}$, we have $\widehat{\mathrm{HF}}\left(-\Sigma\left(L^{+}\right)\right)=$ $\widehat{\mathrm{HF}}\left(S^{3}\right)=\mathbb{Z} / 2 \mathbb{Z}$, and also $\widehat{\mathrm{HF}}\left(-\Sigma\left(L_{1}^{+}\right)\right)=\mathbb{Z} / 2 \mathbb{Z}$, while $\widehat{\mathrm{HF}}\left(-\Sigma\left(L_{0}^{+}\right)\right)=$ $\widehat{\mathrm{HF}}\left(S^{1} \times S^{2}\right)=\mathbb{Z} / 2 \mathbb{Z}_{(-1 / 2)} \oplus \mathbb{Z} / 2 \mathbb{Z}_{(+1 / 2)}$, where the subscripts indicate the gradings. The map $F: \widehat{\mathrm{HF}}\left(-\Sigma\left(L^{+}\right)\right) \rightarrow \widehat{\mathrm{HF}}\left(-\Sigma\left(L_{0}^{+}\right)\right)$of the surgery exact 

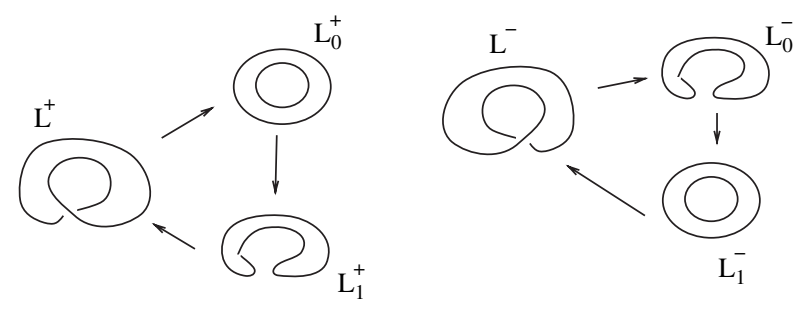

Figure 7. The two unknots.

sequence is the isomorphism between $\mathbb{Z} / 2 \mathbb{Z}$ and $\mathbb{Z} / 2 \mathbb{Z}_{(+1 / 2)} \subset \widehat{\mathrm{HF}}\left(-\Sigma\left(L_{0}^{+}\right)\right)$; the map $D: \widehat{\mathrm{HF}}\left(-\Sigma\left(L_{0}^{+}\right)\right) \rightarrow \widehat{\mathrm{HF}}\left(-\Sigma\left(L_{1}^{+}\right)\right)$is an isomorphism between $\mathbb{Z} / 2 \mathbb{Z}_{(-1 / 2)}$ and $\mathbb{Z} / 2 \mathbb{Z}=\widehat{\mathrm{HF}}\left(-\Sigma\left(L_{1}^{+}\right)\right)$. On the other hand, $\widetilde{\mathrm{Kh}}\left(L^{+}\right)=\mathbb{Z} / 2 \mathbb{Z}$ is generated by a $\mathbf{u}_{+}$, and so is $\widetilde{\mathrm{Kh}}\left(L_{1}^{+}\right)=\mathbb{Z} / 2 \mathbb{Z}$. The group $\widetilde{\mathrm{Kh}}\left(L_{0}^{+}\right)=$ $\mathbb{Z} / 2 \mathbb{Z} \oplus \mathbb{Z} / 2 \mathbb{Z}$ is generated by $\mathbf{u}_{-} \otimes \mathbf{u}_{+}$and $\mathbf{u}_{+} \otimes \mathbf{u}_{+}$. The map $f: \widetilde{\mathrm{Kh}}\left(L^{+}\right) \rightarrow$ $\widetilde{\mathrm{Kh}}\left(L_{0}^{+}\right)$sends $\mathbf{u}_{+}$to $\mathbf{u}_{-} \otimes \mathbf{u}_{+}$, while $d: \widetilde{\mathrm{Kh}}\left(L_{0}^{+}\right) \rightarrow \widetilde{\mathrm{Kh}}\left(L_{1}^{+}\right)$sends $\mathbf{u}_{+} \otimes \mathbf{u}_{+}$ to $\mathbf{u}_{+}$. The groups $\widehat{\mathrm{HF}}\left(-\Sigma\left(L^{+}\right)\right)$and $\widetilde{\mathrm{Kh}}\left(L^{+}\right)$can be obtained as homology of complexes $\left(\widehat{\mathrm{HF}}\left(-\Sigma\left(L_{0}^{+}\right)\right) \oplus \widehat{\mathrm{HF}}\left(-\Sigma\left(L_{1}^{+}\right)\right), D\right)$ and $\left(\widetilde{\mathrm{Kh}}\left(L_{0}^{+}\right) \oplus \widetilde{\mathrm{Kh}}\left(L_{1}^{+}\right), d\right)$, respectively.

We observe:

1) $c\left(\xi_{L^{+}}\right)=1 \in \mathbb{Z} / 2 \mathbb{Z}=\widehat{\mathrm{HF}}\left(-\Sigma\left(L^{+}\right)\right)$, and $\psi\left(L^{+}\right)=\mathbf{u}_{+} \in \mathbb{Z} / 2 \mathbb{Z}=$ $\widehat{\mathrm{Kh}}\left(L^{+}\right)$

2) The maps $F$ and $f$ are associated to a positive crossing resolution, so $F\left(c\left(\xi_{L^{+}}\right)\right)=c\left(\xi_{L_{0}^{+}}\right)=1 \in \mathbb{Z} / 2 \mathbb{Z}_{+1 / 2}, f\left(\psi\left(L^{+}\right)\right)=\psi\left(L_{0}^{+}\right)=\mathbf{u}_{-} \otimes \mathbf{u}_{+} ;$

3) $D\left(c\left(\xi_{L_{0}^{+}}\right)\right)=0=d\left(\psi\left(L_{0}^{+}\right)\right)$;

4) As an element of $\left.\widetilde{\mathrm{Kh}}\left(L_{0}^{+}\right) \oplus \widetilde{\mathrm{Kh}}\left(L_{1}^{+}\right)=\widetilde{C \mathrm{Kh}}(L), \psi\left(L_{0}^{+}\right)\right)=\mathbf{u}_{-} \otimes \mathbf{u}_{+}$ is the element $\tilde{\psi}\left(L^{+}\right)$that represents the class of $\psi\left(L^{+}\right)$. The identity $d\left(\tilde{\psi}\left(L^{+}\right)\right)=0$ means that $\tilde{\psi}\left(L^{+}\right)$is a cycle.

For the link $L^{-}$, we have $\widehat{\mathrm{HF}}\left(-\Sigma\left(L^{-}\right)\right)=\widehat{\mathrm{HF}}\left(S^{3}\right)=\mathbb{Z} / 2 \mathbb{Z}$, and now $\widehat{\mathrm{HF}}\left(-\Sigma\left(L_{0}^{-}\right)\right)=\mathbb{Z} / 2 \mathbb{Z}$, while $\widehat{\mathrm{HF}}\left(-\Sigma\left(L_{1}^{-}\right)\right)=\widehat{\mathrm{HF}}\left(S^{1} \times S^{2}\right)=\mathbb{Z} / 2 \mathbb{Z}_{(-1 / 2)} \oplus$ $\mathbb{Z} / 2 \mathbb{Z}_{(+1 / 2)}$. The map $F: \widehat{\mathrm{HF}}\left(-\Sigma\left(L_{1}^{-}\right)\right) \rightarrow \widehat{\mathrm{HF}}\left(-\Sigma\left(L^{-}\right)\right)$of the surgery exact sequence is the isomorphism between $\mathbb{Z} / 2 \mathbb{Z}_{(-1 / 2)} \subset \widehat{\mathrm{HF}}\left(-\Sigma\left(L_{1}^{-}\right)\right)$and $\mathbb{Z} / 2 \mathbb{Z}=\widehat{\mathrm{HF}}\left(-\Sigma\left(L^{-}\right)\right)$. The map $D: \widehat{\mathrm{HF}}\left(-\Sigma\left(L_{0}^{-}\right)\right) \rightarrow \widehat{\mathrm{HF}}\left(-\Sigma\left(L_{1}^{-}\right)\right)$is an isomorphism between $\mathbb{Z} / 2 \mathbb{Z}=\widehat{\mathrm{HF}}\left(-\Sigma\left(L_{0}^{-}\right)\right)$and $\mathbb{Z} / 2 \mathbb{Z}_{(+1 / 2)}$. The Khovanov homology $\widetilde{\mathrm{Kh}}\left(L^{-}\right)=\mathbb{Z} / 2 \mathbb{Z}$ is generated by a $\mathbf{u}_{+}$, and so is $\widetilde{\mathrm{Kh}}\left(L_{0}^{-}\right)=\mathbb{Z} / 2 \mathbb{Z}$, while $\widetilde{\mathrm{Kh}}\left(L_{1}^{-}\right)=\mathbb{Z} / 2 \mathbb{Z} \oplus \mathbb{Z} / 2 \mathbb{Z}$ is generated by $\mathbf{u}_{-} \otimes \mathbf{u}_{+}$and $\mathbf{u}_{+} \otimes \mathbf{u}_{+}$. The map $f: \widetilde{\mathrm{Kh}}\left(L_{1}^{-}\right) \rightarrow \widetilde{\mathrm{Kh}}\left(L^{-}\right)$sends $\mathbf{u}_{+} \otimes \mathbf{u}_{+}$to $\mathbf{u}_{+}$, and $d: \widetilde{\mathrm{Kh}}\left(L_{0}^{-}\right) \rightarrow \widetilde{\mathrm{Kh}}\left(L_{1}^{-}\right)$ 
sends $\mathbf{u}_{+}$to $\mathbf{u}_{-} \otimes \mathbf{u}_{+}$. In this case, we have:

1) $c\left(\xi_{L^{-}}\right)=0$, and $\psi\left(L^{-}\right)=0$;

2) The maps $F$ and $f$ are associated to a positive crossing resolution, so $F\left(c\left(\xi_{L_{1}^{-}}\right)\right)=c\left(\xi_{L^{-}}\right)=0, f\left(\psi\left(L_{1}^{-}\right)\right)=\psi\left(L^{-}\right)=0 ;$

3) The element $c\left(\xi_{L_{1}^{-}}\right)=1 \in \mathbb{Z} / 2 \mathbb{Z}_{+1 / 2}$ is the image under $D$ of the generator of $\widehat{\mathrm{HF}}\left(-\Sigma\left(L_{0}^{-}\right) ; \psi\left(L_{1}^{-}\right)\right.$is the image of $\mathbf{u}_{+} \in \widetilde{\mathrm{Kh}}\left(L_{0}^{-}\right)$under $d$. As an element of $\widetilde{C \mathrm{Kh}}\left(L^{-}\right), \psi\left(L_{1}^{-}\right)$is precisely $\tilde{\psi}\left(L^{-}\right)$, and the identity $\tilde{\psi}\left(L^{-}\right)=d\left(\mathbf{u}_{+}\right)$means that the invariant $\psi\left(L^{-}\right)$vanishes in $\widetilde{\mathrm{Kh}}\left(L^{-}\right)$.

Proof of Theorem 1.6. Let the transverse link be represented by a braid $L$ whose diagram is alternating. First of all, we observe that such a braid enjoys little freedom. Indeed, suppose there is a factor of $\sigma_{1}$ in the braid word. This gives a positive crossing in the diagram, which means that the "neighboring" crossing must be negative. Considering the crossings one by one, we can conclude that all factors of $\sigma_{1}$ in the braid word come with positive exponents, all factors of $\sigma_{2}$ come with negative exponents (that is, the braid word contains $\sigma_{2}^{-1}$ 's but no $\sigma_{2}$ 's), all factors for $\sigma_{3}$ again have positive exponents, and so on. Proposition 6.8 and the analogous proposition of [10] imply that $c\left(\xi_{L}\right)=0$ and $\psi(L)=0$ unless the braid word for $L$ contains no $\sigma_{2 k}$ for any $k>0$. The latter situation means that $L$ is simply a disjoint union of $\left(2, n_{i}\right)$ torus links, and this is the only case where the theorem needs proof.

When $L=T(2, n)$ is given by a 2 -braid with $n$ positive crossings, both $c\left(\xi_{L}\right)$ and $\psi(L)$ are non-zero. The map $f$ is associated to the resolution of a positive crossing; by definition, $\psi(L)$ is a homogeneous element of homological degree $0[\mathbf{1 0}]$. Recall that Khovanov homology $\widetilde{\operatorname{Kh}}(T(2, n))$ has a special form [23], so that in every homological degree the component of $\widetilde{\mathrm{Kh}}(T(2, n))$ is at most one-dimensional; we can then say that $\psi(L)$ is the (unique) element of the lowest homological grading in $\widetilde{\mathrm{Kh}}(L)$. Also, $\widehat{\mathrm{HF}}(-\Sigma(L))=(\mathbb{Z} / 2 \mathbb{Z})^{n}$ since $\Sigma(L)$ is the lens space $-L(n, 1)$. When $n=1$, it follows that $c\left(\xi_{L}\right)=c_{0}\left(\xi_{L}\right)=\psi(L)$.

When $n>1$, we proceed by induction. Consider the link $L=T(2, n+1)$ and its two resolutions $L_{0}=T(2, n)$ and $L_{1}=$ unknot (Figure 8).

For these three links, (7.1) becomes

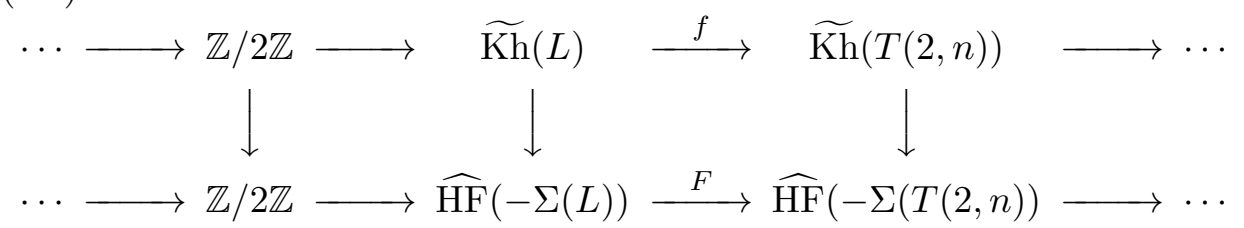

where $\mathbb{Z} / 2 \mathbb{Z}$ is the homology of the unknot. 


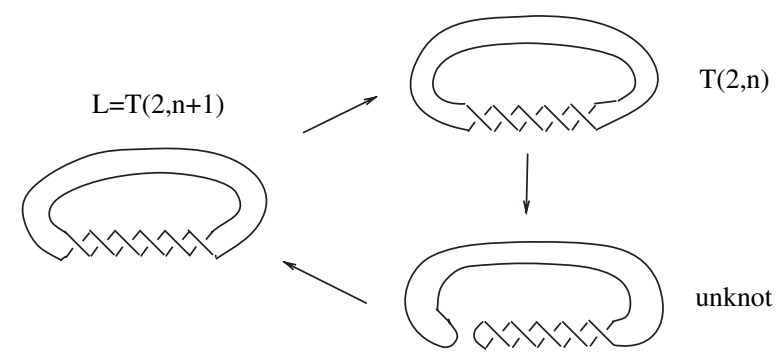

Figure 8. The torus link $L$ and its resolutions.

The image of $\mathbb{Z} / 2 \mathbb{Z}$ in $\widetilde{\mathrm{Kh}}(L)$ lies in the homological degree higher than the minimal degree 0 (where $\psi(L)$ lives). Then, in the lower row of the above diagram, the image of $\mathbb{Z} / 2 \mathbb{Z}$ in the associated graded group of $\widehat{\mathrm{HF}}(-\Sigma(L))$ also lies in the higher filtration degree. The map $F$ on $\widehat{\operatorname{HF}}(-\Sigma(L))$ is induced by the resolution of a positive crossing, so $F\left(c\left(\xi_{L}\right)\right)=c\left(\xi_{T(2, n)}\right)$. By the induction hypothesis, $c_{0}\left(\xi_{T(2, n)}\right)$ lies in the lowest degree subquotient of $\widehat{\mathrm{HF}}(-\Sigma(T(2, n)))$ and agrees with $\psi(T(2, n))$. The map $F$ preserves the filtration; it follows that it must send the lowest degree subquotient of $\widehat{\mathrm{HF}}(-\Sigma(L))$ to the lowest degree subquotient of $\widehat{\mathrm{HF}}(-\Sigma(T(2, n)))$, since otherwise we could not have $F\left(c\left(\xi_{L}\right)\right)=c\left(\xi_{T(2, n)}\right)$. Then, $c_{0}\left(\xi_{L}\right)$ is also non-trivial, and so $c_{0}\left(\xi_{L}\right)=\psi(L)$.

It remains to deal with the case when $L$ is a disjoint union of torus links. Let $T_{1}=T\left(2, n_{1}\right), T_{2}=T\left(2, n_{2}\right)$, and $L=T_{1} \sqcup T_{2}$. Then, the contact manifold $\left(\Sigma(L), \xi_{L}\right)$ is the connected sum of $\left(\Sigma\left(T_{1}\right), \xi_{T_{1}}\right)$, $\left(\Sigma\left(T_{2}\right), \xi_{T_{2}}\right)$, and $\left(S^{1} \times S^{2}, \xi_{0}\right)$. Then, $\widehat{\mathrm{HF}}(-\Sigma(L))=\widehat{\mathrm{HF}}\left(-\Sigma\left(T\left(2, n_{1}\right)\right)\right) \otimes$ $\widehat{\mathrm{HF}}\left(-\Sigma\left(T\left(2, n_{2}\right)\right)\right) \otimes \widehat{\mathrm{HF}}\left(-S^{1} \times S^{2}\right)$, and $c\left(\xi_{L}\right)=c\left(\xi_{T_{1}}\right) \otimes c\left(\xi_{T_{2}}\right) \otimes c\left(\xi_{0}\right)$, where $c\left(\xi_{0}\right) \in \widehat{\mathrm{HF}}\left(-S^{1} \times S^{2}\right)=\mathbb{Z} / 2 \mathbb{Z}_{(-1 / 2)} \oplus \mathbb{Z} / 2 \mathbb{Z}_{(+1 / 2)}$ is the generator of $\mathbb{Z} / 2 \mathbb{Z}_{(+1 / 2)}$. Similarly, for the Khovanov homology, we have $\widetilde{\mathrm{Kh}}(L)=\widetilde{\mathrm{Kh}}\left(T_{1}\right) \otimes K h\left(T_{2}\right)=\widetilde{\mathrm{Kh}}\left(T_{1}\right) \otimes \widetilde{\mathrm{Kh}}\left(T_{2}\right) \otimes(\mathbb{Z} / 2 \mathbb{Z} \oplus \mathbb{Z} / 2 \mathbb{Z})$, and $\psi(L)=\psi\left(T_{1}\right) \otimes \psi\left(T_{2}\right) \otimes \mathbf{u}_{-}$. The correspondence $\psi(L)=c_{0}\left(\xi_{L}\right)$ follows, since the isomorphism between the homology groups maps the generator of $\mathbb{Z} / 2 \mathbb{Z}_{(+1 / 2)}$ to the $\mathbf{u}_{-}$.

\section{Acknowledgements}

I would like to thank Peter Ozsváth and Andras Stipsicz for very helpful discussions. I am also very grateful for the referee's remarks and suggestions.

\section{References}

[1] J. Etnyre and K. Honda, Knots and contact geometry. I. Torus knots, and the figure eight knot, J. Symplectic Geom. 1(1) (2001), 63-120. 
[2] J. Birman and B. Menasco, Stabilization in the braid groups-II: transversal simplicity of knots, math.GT/0310280.

[3] J. Etnyre and K. Honda, Cabling and transverse simplicity, math.SG/0306330.

[4] E. Giroux, Géométrie de contact: de la dimension trois vers les dimensions supérieures, Proceedings of the International Congress of Mathematicians, II (Beijing, 2002), Higher Ed. Press, Beijing, 2002, 405-414.

[5] F. Ding and H. Geiges, A Legendrian surgery presentation of contact 3-manifolds, Math. Proc. Cambridge Philos. Soc. 136(3) (2004), 583-598.

[6] F. Ding, H. Geiges and A. Stipsicz, Surgery diagrams for contact 3-manifolds, Turkish J. Math. 28(1), (2004), 41-74.

[7] R. Gompf, Handlebody construction of Stein surfaces, Ann. Math. (2) 148(2) (1998), 619-693.

[8] P. Oszváth and Z. Szabó, Holomorphic disks and topological invariants for closed 3-manifolds, Ann. Math. (2) 159(3) (2004), 1027-1158.

[9] P. Oszváth and Z. Szabó, Heegaard Floer homologies and contact structures, Duke Math. J. 129(1) (2005), 39-61.

[10] O. Plamenevskaya, Transverse knots and Khovanov homology, preprint.

[11] P. Ozsváth and Z. Szabó, On the Heegaard Floer homology of branched double-covers, Adv. Math. 194(1) (2005), 1-33.

[12] D. Bar-Natan, On Khovanov's categorification of the Jones polynomial, Algebr. Geom. Topol. 2 (2002), 337-370.

[13] A. Shumakovich, KhoHo pari package, 2003, available at: www.geometrie.ch/KhoHo/

[14] K. Honda, On the classification of tight contact structures. I, Geom. Topol. 4 (2000), 309-368.

[15] J. Etnyre, Legendrian and Transversal Knots, math.SG/0306256.

[16] D. Bennequin, Entrelacements et équations de Pfaff, Astérisque 107, 108 (1983), $87-161$.

[17] L. Rudolph, Quasipositivity as an obstruction to sliceness, Bull. Amer. Math. Soc. 29(1) (1993), 51-59.

[18] J. Etnyre, Transversal torus knots, Geom. Topol. 3 (1999), 253-268.

[19] J. Birman, Braids, links and mapping class groups, Annals of Mathematical Studies 82 Princeton Univ. Press, Princeton, NJ, 1974.

[20] P. Lisca and A. Stipsicz, Seifert fibered contact three-manifolds via surgery, Algebr. Geom. Topol. 4 (2004), 199-217.

[21] J. Etnyre and K. Honda, On connected sums and Legendrian knots, Adv. Math. 179(1) (2003), 59-74.

[22] J. Rasmussen, Khovanov homology and the slice genus, math.GT/0402131.

[23] M. Khovanov, A categorification of the Jones polynomial, Duke Math. J. 101(3) (2000), 359-426.

Department of Mathematics

SUNY STONY BROOK

STONY BRoOK, NY 11794-3651

E-mail address: olga@math.sunysb.edu

Received 26 September 2005 